\title{
Ocorrência de Lecanodiaspis dendrobii (Hemiptera: Lecanodiaspididae) e do parasitoide associado Cephaleta sp. (Hymenoptera: Pteromalidae) em mogno africano no Brasil
}

\author{
Occurrence of Lecanodiaspis dendrobii (Hemiptera: Lecanodiaspididae) and the \\ associated parasitoid Cephaleta sp. (Hymenoptera: Pteromalidae) in african \\ mahogany in Brazil
}

\section{Edgley Soares da Silva', Mauricio Lourenzoni Augusti', Roberto Dantas de Medeiros"I, José de Anchieta Alves de Albuquerque ${ }^{\mathrm{III}}$, Raphael Henrique da Silva Siqueira ${ }^{\mathrm{IV}}$, Antônio Cesar Silva Lima ${ }^{\mathrm{III}}$}

\begin{abstract}
Resumo
A expansão do cultivo de mogno africano no Brasil, assim como outras espécies exóticas, remete ao surgimento de pragas e doenças até então não relatadas na literatura. Nesse sentido, amostras suspeitas de conter uma nova espécie de cochonilha atacando a casca, ramos e hastes de plantas de mogno africano e parasitadas por um microhimenóptero, foram coletadas em um plantio de dez hectares, com cinco anos de idade em uma fazenda no município de Cantá, Roraima, Brasil. O material amostrado continha cochonilhas lecanodiaspidídeos, em todas as fases de desenvolvimento, ovos, ninfas e adultos, machos e fêmeas, as quais foram identificadas como Lecanodiaspis dendrobii Douglas, 1892 (Hemiptera: Lecanodiaspididae). O parasitoide associado foi identificado como Cephaleta sp. (Hymenoptera: Pteromalidae) apresentando grande potencial no controle biológico de Lecanodiaspis dendrobii. Este é o primeiro registro de ataque da cochonilha Lecanodiaspis dendrobii (Hemiptera: Lecanodiaspididae) e do parasitoide associado Cephaleta sp. (Hymenoptera: Pteromalidae) em mogno africano no Brasil.
\end{abstract}

Palavras-chave: Khaya ivorensis; Entomologia florestal; Inseto-praga

\begin{abstract}
The expansion of African mahogany cultivation in Brazil, as well as other exotic species, refers to the appearance of pests and diseases previously unreported in the literature. In this sense, samples suspected of containing a new species of cochineal species attacking the bark, branches and stems of African mahogany plants and parasitized by a microhimenopter, were collected in a ten hectare plantation with five years of age on a farm in the municipality of Cantá, Roraima state, Brazil. The sampled material contained lecanodiaspididae in all stages of development, eggs, nymphs and adults, both male and female, which were identified as Lecanodiaspis dendrobii Douglas, 1892 (Hemiptera: Lecanodiaspididae). The associated parasitoid was identified as Cephaleta sp. (Hymenoptera: Pteromalidae) presenting great potential in the biological control of Lecanodiaspis dendrobii. This is the first attack record of Lecanodiaspis dendrobii (Hemiptera: Lecanodiaspididae) and the associated parasitoid Cephaleta sp. (Hymenoptera: Pteromalidae) in African mahogany in Brazil.
\end{abstract}

Keywords: Khaya ivorensis; Forest entomology; Insect pest

Engenheiro Agrônomo, Doutorando do Programa de Pós-Graduação em Agronomia, Centro de Ciências Agrárias, Universidade Federal de Roraima, BR 174, Km 12, Monte Cristo, CEP 69310-270, Boa Vista (RR), Brasil. edgleyrr@gmail.com (ORCID: 0000-0003-4628-1920) / mauricioaugusti86@ gmail.com (ORCID: 0000-0001-8441-7361)

II Engenheiro Agrônomo, Dr., Pesquisador da Empresa Brasileira de Pesquisa Agropecuária, Centro de Pesquisa Agroflorestal de Roraima, BR 174, km 8, Distrito Industrial, CEP 69301-970, Boa Vista (RR), Brasil. roberto.medeiros@embrapa.br (ORCID: 0000-0002-5601-049X)

III Engenheiro Agrônomo, Dr., Professor do Programa de Pós-Graduação em Agronomia, Centro de Ciências Agrárias, Universidade Federal de Roraima, BR 174, Km 12, Monte Cristo, CEP 69310-270, Boa Vista (RR), Brasil. anchietaufrr@gmail.com (ORCID: 0000-0003-4391-258X) / cesar. lima@ufrr.br (ORCID: 0000-0002-1164-1589)

IV Engenheiro Agrônomo, Dr., Professor do Instituto Federal de Educação, Ciência e Tecnologia de Roraima, Campus Amajari, Rodovia Antonino Menezes da Silva, Km 3, CEP 69343-000, Amajari (RR), Brasil. raphael.siqueira@ifrr.edu.br (ORCID: 0000-0002-9797-5992) 


\section{Introdução}

O mogno africano (Khaya ivorensis A. Chevalier) pertencente à família Meliaceae, mesma família do mogno brasileiro (Swietenia macrophylla King), da andiroba (Carapa guianensis Aubl.) e do cedro (Cedrela fissilis Vell.), é nativo das planícies tropicais úmidas da África Ocidental, onde forma extensos bosques no Congo, Guiné, Costa do Marfim, Gana, Togo, Benin, Nigéria e Camarões (ALVES JÚNIOR et al., 2016; RIBEIRO; FERRAZ FILHO; SCOLFORO, 2017). A espécie possui grande importância econômica, devido às características tecnológicas e estéticas de sua madeira, empregada na indústria moveleira, naval, construção civil e na confecção de painéis, laminados, adornos, pisos e lambris (PINHEIRO et al., 2011).

A espécie Khaya ivorensis foi introduzida no Brasil na década de 1970, na região Norte, com o objetivo de substituir o mogno brasileiro, intensamente explorado pelo setor madeireiro na época e sob risco de extinção. A espécie rapidamente se adaptou as mais variadas condições do território brasileiro, sobretudo, devido às características de clima, temperatura e pluviosidade serem semelhantes as de seu centro de origem (CASAROLI et al., 2018).

A rusticidade do mogno africano, aliada ao elevado preço de sua madeira, nos mercados nacional e internacional, gerou atratividade econômica e levou a investimentos em plantios comerciais em todo o Brasil, onde atualmente a área plantada já ultrapassa 10 mil hectares, com perspectiva de aumento (RIBEIRO; FERRAZ FILHO; SCOLFORO, 2017).

Além do rápido crescimento e da maior facilidade na produção de mudas (CORCIOLI; BORGES; JESUS, 2016), a principal vantagem do mogno africano em relação ao brasileiro se deu pela sua resistência à broca do ponteiro, Hypsipyla grandella Zeller (Lepidoptera: Pyralidae), principal praga de Swietenia macrophylla (DIONISIO et al., 2016; KLEIN et al., 2016; CASTRO; MONTALVÃO; MONNERAT, 2018). Porém, vale salientar que já há relatos no Brasil do ataque da referida broca em Khaya ivorensis (ZANETTI et al., 2017; LEMES et al., 2019).

Além da Hypsipyla grandella, as únicas espécies registradas causando danos em mogno africano no Brasil; são: formigas cortadeiras do gênero Atta spp. (Hymenoptera: Formicidae), abelhas arapuá, Trigona spinipes e Trigona hyalinata (Hymenoptera: Apidae) (KLEIN et al., 2016), broca do pecíolo, Xyleborus spp. (Coleóptero: Escolytinae) e os ácaros Mononychellus sp. e Oligonychus sp. (Arachnida: Acari) (NASCIMENTO et al., 2016).

Segundo Perez et al. (2010), o baixo número de espécies relacionadas ao mogno africano se deve, sobretudo, a produção de metabólitos secundários, que atuam como bioativos contra a ação de insetos. No entanto, Casaroli et al. (2018) salientam que a expansão do cultivo de mogno africano pelo Brasil, implica na exposição dessa espécie a variadas condições climáticas e ambientais, remetendo ao surgimento de pragas e doenças até então não relatadas.

A cochonilha é um inseto-praga presente e relatado nas mais diversas regiões brasileiras. Caracteriza-se pelo seu hábito gregário e por serem extremamente prolíferas. O principal prejuízo causado por esse inseto deve-se à contínua sucção de seiva, o que leva ao enfraquecimento das plantas. Além disso, as substâncias excretadas (honeydew) por essa praga promovem um ambiente favorável à proliferação de fungos causadores de fumagina, acarretando em redução da fotossíntese (SANTOS; PERRONTI, 2017).

Menções quanto aos prejuízos causados por cochonilhas no Brasil, remetem-se, principalmente aos cultivos de hortaliças, frutíferas e ornamentais, no entanto, há relatos desses insetos infestando também algumas espécies florestais, sobretudo, eucalipto (Eucalyptus spp) (SANTOS et al., 2008), acácia (Acacia mangium) (MARSARO JÚNIOR et al., 2016) e teca (Tectona grandis) (PERES-FILHO et al., 2017).

Nesse sentido, o objetivo deste estudo foi registrar a primeira ocorrência da cochonilha Lecanodiaspis dendrobii Douglas, 1892 (Hemiptera: Lecanodiaspididae) e do parasitoide associado Cephaleta sp. (Hymenoptera: Pteromalidae), em mogno africano no Brasil. 


\section{Material e métodos}

Amostras suspeitas de conter uma nova espécie de cochonilha atacando a casca, ramos e hastes de plantas de mogno africano e parasitadas por um microhimenóptero foram coletadas em junho de 2019, em um plantio de dez hectares, com cinco anos de idade, cultivado em espaçamento 6,0 $\mathrm{m}$ x 6,0 m, em consórcio com banana (Musa sp.) e açaí (Euterpe oleracea Mart.), localizado em uma fazenda no município de Cantá, Roraima, Brasil a $02^{\circ} 28^{\prime} 46^{\prime \prime} \mathrm{N}$ e $60^{\circ} 34^{\prime} 41^{\prime}$ O.

A área amostrada pertence ao sistema ecológico das florestas densas da região amazônica, sob influência do clima do tipo Am, quente e semiúmido, segundo classificação de Köppen, com estação chuvosa de abril a setembro e estação seca de outubro a março, caracterizado por médias anuais de precipitação, umidade relativa e temperatura ambiente de $2.090 \mathrm{~mm}, 70 \%$ e $27,4^{\circ} \mathrm{C}$, respectivamente (FERREIRA; TONINI, 2009).

Os danos nas plantas foram fotografados e o material amostrado (ramos, cascas e hastes) foi transportado em sacos plásticos para o Laboratório de Entomologia da Embrapa Roraima, Boa Vista, Roraima, onde parte dos espécimes foi contado e acondicionado em solução de álcool $70 \%$, para posterior identificação da espécie e a outra parte foi colocada em recipiente de vidro de $150 \mathrm{~mL}$, coberto com tela fina (malha de $2 \mathrm{~mm}$ ), para posterior emergência do parasitoide.

As cochonilhas foram examinadas por um entomologista da Embrapa Roraima, sob um estereomicroscópio, seguindo a metodologia descrita por Gullan (1984) e identificadas de acordo com as características morfológicas da fêmea adulta como descrita por Miller, Rung e Parikh (2014). Para identificação do microhimenóptero associado à cochonilha utilizou-se a metodologia de Bouček e Heydon (1997) em nível de gênero. Os exemplares de ambas as espécies encontramse depositados na coleção entomológica do Laboratório de Entomologia da Embrapa Roraima.

\section{Resultados e discussão}

As amostras continham cochonilhas lecanodiaspidídeos, em todas as fases de desenvolvimento do hemíptera, isto é, ovos, ninfas e adultos, machos e fêmeas (Figura 1A), as quais foram identificadas como Lecanodiaspis dendrobii Douglas, 1892 (Hemiptera: Lecanodiaspididae). Esse é o primeiro relato de ataque dessa espécie em mogno africano (Khaya ivorensis) no Brasil.

Em uma área de dez hectares, onde o levantamento foi realizado, verificou-se a infestação de Lecanodiaspis dendrobii em 15\% das plantas, com 35\% de morte. As plantas infestadas pela referida espécie apresentavam definhamento progressivo, com rachaduras e excreções no caule, seguido de morte dos ramos apicais (Figura 1B). O melado excretado (honeydew) pelas cochonilhas, sobre as folhas e caule, favoreceu o desenvolvimento de fumagina, comprometendo a fotossíntese, causando queda prematura das folhas e morte da planta. 
Silva, E. S.; Augusti, M. L.; Medeiros, R. D.; Albuquerque, J. A. A.;

Siqueira, R. H. S.; Lima, A. C. S.

Figura 1 - A: infestação de machos e fêmeas de diferentes instares de Lecanodiaspis dendrobii; B: Danos em plantas de mogno africano (Khaya ivorensis A. Chevalier), causados por Lecanodiaspis dendrobii

Figure 1 - A: infestation of males and females of different instars of Lecanodiaspis dendrobii; B: Damage in African mahogany (Khaya ivorensis A. Chevalier) plants, caused by Lecanodiaspis dendrobii

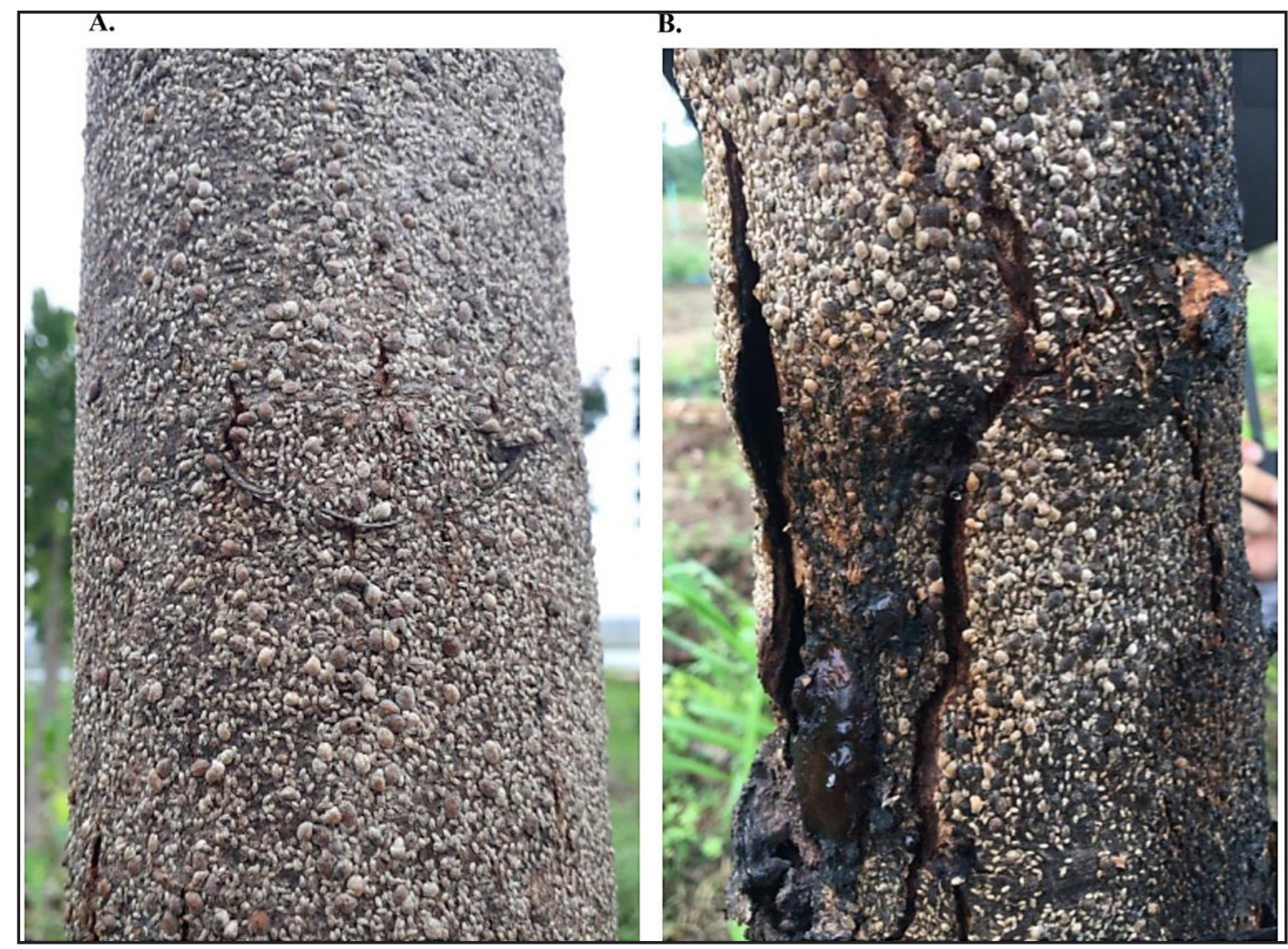

Fonte: Autores (2019)

A Lecanodiaspis dendrobii é um inseto de escama, polífago, que se alimenta de muitas espécies de plantas, distribuídas, até o momento, em oito famílias botânicas: Anacardiaceae, Annonaceae, Euphorbiaceae, Fabaceae, Moraceae, Orchidaceae, Rutaceae e Verbenaceae (GARCÍA MORALES et al., 2015; MARSARO JÚNIOR et al., 2016).

Macroscopicamente, o corpo da fêmea adulta de Lecanodiaspis dendrobii é acastanhado, achatado, coberto por um teste castanho claro infundido com cera branca secundária; teste com carina longitudinal médio dorsal cruzada por linhas levantadas transversais. Teste masculino semelhante em textura e aparência, porém, mais alongado. Microscopicamente, Lecanodiaspis dendrobii é caracterizado por ter 3 a 5 placas cribriformes de cada lado do corpo; cerdas espirais anteriores e posteriores presentes; sulcos espiracular posteriores divididos; antenas de 7 a 9 segmentos e pernas rudimentares (MILLER; RUNG; PARIKH, 2014).

A Lecanodiaspis dendrobii foi descrita por Douglas em 1892, coletada em Dendrobium calceolaria em Demerara, República da Guiana (DOUGLAS, 1892). Mais tarde foi relatada em vários países do continente americano: Argentina, Colômbia, Guatemala, Honduras, Uruguai, Venezuela e México (GARCÍA MORALES et al., 2015). Recentemente a Lecanodiaspis dendrobii foi registrada pela primeira vez no Brasil, no Estado de Roraima, infestando Acacia mangium W., Xylopia aromatica L., Leucaena leucocephala L., Morus nigra L. Citrus reticulata B., Tectona grandis 
L., Anacardium occidentale L. e Annona squamosa L. Contudo, os danos mais significativos foram registrados em Acacia mangium, Morus nigra e Leucaena leucocephala (MARSARO JÚNIOR et al., 2016).

No Brasil, já foram relatadas algumas espécies de cochonilha infestando mogno, porém os registros remetem-se ao mogno brasileiro (S. macrophylla) e tratam-se das espécies Saissetia miranda (Cockerell e Parrott) (Hemiptera: Coccidae) (CASTRO et al., 2018) e Phenacoccus solenopsis Tinsley (Hemiptera: Pseudococcidae) (CASTRO et al., 2019), não havendo até o momento relatos para o mogno africano.

O parasitoide associado à Lecanodiaspis dendrobii foi identificado como Cephaleta sp. (Hymenoptera: Pteromalidae) (Figura 2A). Até o presente momento os inimigos naturais de Lecanodiaspis dendrobii conhecidos são os endoparasitóides gregários Anisophleps alternata F. e Metaphycus terani F. (Hymenoptera: Encyrtidae) (FIDALGO, 1981), Signiphora (Hymenoptera: Signiphoridae) (Blanchard,1938), Marietta caridei B. (Hymenoptera: Aphelinidae) (DE SANTIS, 1967) e Cephaleta sp. (Hymenoptera: Pteromalidae) (MARSARO JÚNIOR et al., 2016).

\section{Figura 2 - A: Fêmea adulta de Cephaleta sp.; B: Lecanodiaspis dendrobii parasitadas mostrando o orifício de emergência do parasitoide}

Figure 2 - A: Adult female of Cephaleta sp.; B:Lecanodiaspis dendrobii parasitized showing the emergency orifice of the parasitoid

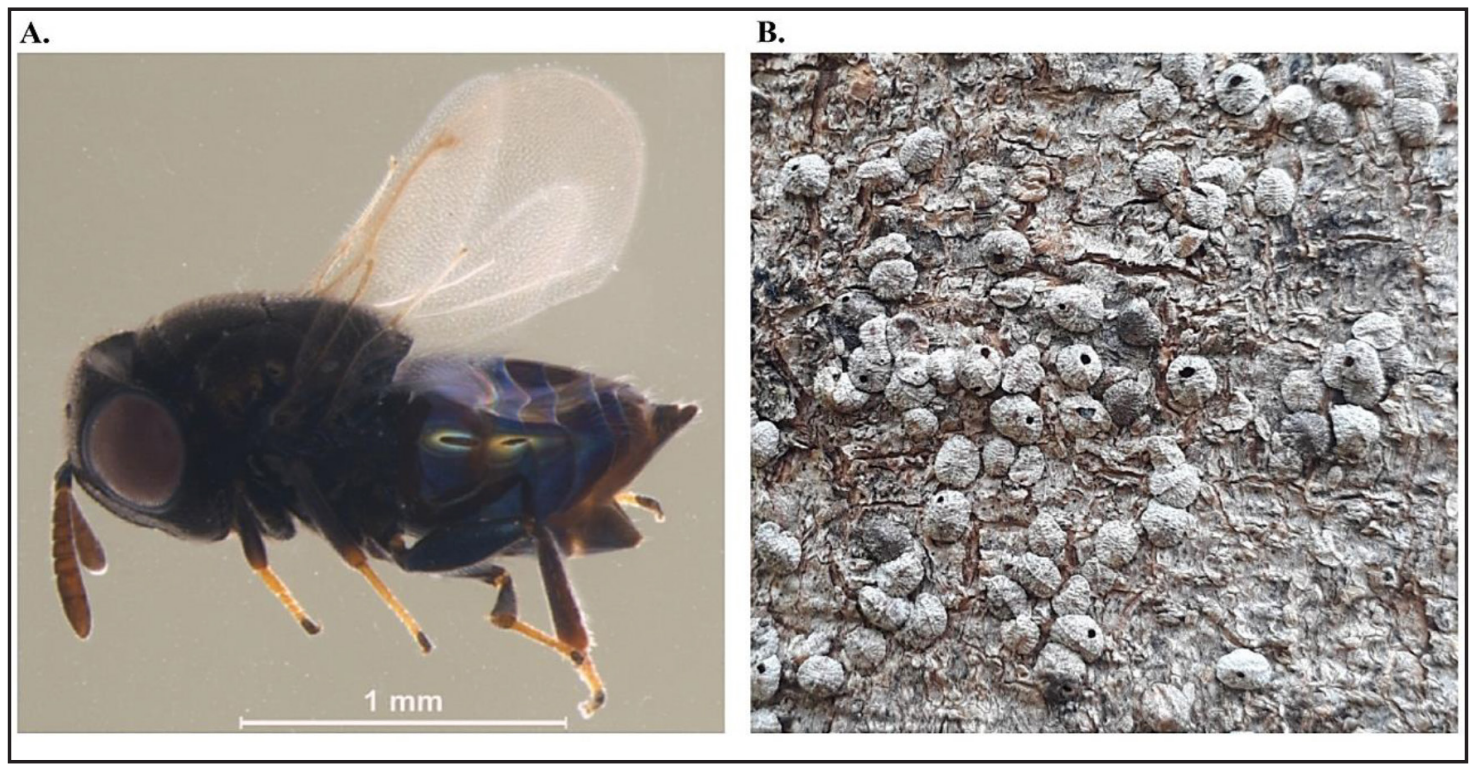

Fonte: A: Marsaro Júnior et al. (2016); B: Autores (2019)

No estado de Roraima, apenas o parasitoide Cephaleta sp. (Hymenoptera: Pteromalidae) foi encontrado em associação com Lecanodiaspis dendrobii em mogno africano. Confirmando a suspeita de Marsaro Júnior et al. (2016), que acreditaram-se tratar de uma nova espécie de Cephaleta encontrada no Brasil, dada as diferenças de Cephalet abrasiliensis, única espécie do gênero conhecida no país.

O potencial de Cephaleta sp. no controle de Lecanodiaspis dendrobii pode ser observado na Figura 2B, onde as lecanodiaspidídeas parasitadas mostram o orifício de saída do parasitoide. No entanto, para que haja uma recomendação de uso da Cephaleta sp. como alternativa de controle biológico de Lecanodiaspis dendrobii, sua atuação parasitária precisa ser melhor elucidada.

Como não existem inseticidas registrados para o mogno africano no Brasil, algumas estratégias de controle de Lecanodiaspis dendrobii podem ser empregadas. Deve-se realizar 
monitoramento e inspeção do plantio, removendo e eliminando os insetos de plantas infestadas, erradicar as plantas quando a infestação for alta e evitar o transporte de mudas ou parte de plantas infestadas, para lugares livres desse inseto, haja visto que sua dispersão em longas distâncias é feita principalmente através de plantas hospedeiras.

\section{Conclusão}

Este é o primeiro registro da cochonilha Lecanodiaspis dendrobii Douglas, 1892 (Hemiptera: Lecanodiaspididae) e do parasitóide associado Cephaleta sp. (Hymenoptera: Pteromalidae) em mogno africano no Brasil.

\section{Referências}

ALVES JÚNIOR, J. et al. Crescimento de mogno africano submetido a diferentes níveis de irrigação por microaspersão. Irriga, Botucatu, v. 21, n. 3, p. 466-480, 2016.

BLANCHARD, E. E. Informaciones técnicas. Boletín Informativo de la Dirección de Sanidad Vegetal, v. 1, n. 1, p. 25-32, 1938.

BOUČEK, Z.; HEYDON, S. L. Pteromalidae. In: GIBSON, G. A. P.; HUBER, J. T.; WOOLLEY, J. B. Annotated keys to the genera of Nearctic Chalcidoidea (Hymenoptera). Ottawa: National Research Council of Canada, 1997. p. 541-692.

CASAROLI, D. et al. Aptidão edafoclimática para o mogno africano no Brasil. Ciência Florestal, Santa Maria, v. 28, n. 1, p. 357-368, 2018.

CASTRO, M. T. et al. First Report of Saissetia miranda (Cockerell e Parrott) (Hemiptera: Coccidae) in Brazil: Occurrence on Mahogany Seedlings, Florida Entomologist, Gainesville, v. 101, n. 2, p. 324-326, 2018.

CASTRO, M. T. et al. Ocorrência da cochonilha Phenacoccus solenopsis Tinsley (Hemiptera: Pseudococcidae) em mudas de mogno (Swietenia macrophylla King). EntomoBrasilis, Vassouras, v. 12, n. 1, p. 41-43, 2019.

CASTRO, M. T.; MONTALVÃO, S. C. L.; MONNERAT, R. G. Damage in fruits of mahogany caused by Hypsipyla grandella (Zeller) (Lepidoptera: Pyralidae) in Brasília, Brazil. EntomoBrasilis, Vassouras, v. 11, n. 2, p. 9-12, 2018.

CORCIOLI, G.; BORGES, J. D.; JESUS, R. P. Deficiência de macro e micronutrientes em mudas maduras de Khaya ivorensis estudadas em viveiro. Revista Cerne, Lavras, v. 22, n. 1, p. 131-128, 2016.

DIONISIO, L. F. S. et al. Ocorrência de Hypsipyla grandella Zeller (Lepidoptera, Pyralidae) no Sul do Estado de Roraima. EntomoBrasilis, Vassouras, v. 9, n. 2, p. 97-100, 2016.

DE SANTIS, L. Catálogo de los Himenópteros Argentinos de la Serie Parasitica, incluyendo Bethyloidea. La Plata: Comision de Investigacion Cientifica, 1967. 337 p.

DOUGLAS, J. W. Notes on some British and exotic Coccidae. Entomologist's Monthly Magazine, London, v. 28, n. 2, p. 207-209, 1892. 
FERREIRA, L. M. M.; TONINI, H. Comportamento da castanha-do-brasil (Bertholletia excelsa) e da cupiúba (Goupia glabra) em sistema agrosilvicultural na região da Confiança, Cantá - Roraima. Acta Amazonica, Manaus, v. 39, n. 4, p. 835-842, 2009.

FIDALGO, A. P. Sobre un nuevo genero y dos nuevas especies de Encirtidos (Hymenoptera: Chalcidoidea) parasitoides de Lecanodiaspis dendrobii Dougl. (Homoptera: Coccoidea) sobre citrus en Tucuman, Argentina. Acta Zoológica Lilloana, Tucuman, v. 36, n. 2, p. 37-43, 1981.

GARCIA MORALES, M. et al. ScaleNet: a literature-based model of scale insect biology and systematics. Database, Oxford, v. 2016, n. 1, p. 1-5, 2016.

GULLAN, P. J. A revision of the gall-forming coccoid genus Apiomorpha Rübsaamen (Homoptera: Eriococcidae: Apiomorphinae). Australian Journal of Zoology, Melbourne, v. 97, n. 1, p. 1-203, 1984.

KLEIN, D. R. et al. General and silvicultura aspects of Cordia americana, Aspidosperma polyneuron, Toona ciliata e Khaya spp. Journal of Agroveterinary Sciences, Lages, v. 15, n. 2, p. 155-164, 2016.

LEMES, P. G. et al. Hypsipyla grandella (Lepidoptera: Pyralidae) boring Khaya ivorensis (Meliaceae) fruits and seeds in Brazil: first report. Florida Entomologist, Gainesville, v. 102, n. 1, p. 266-269, 2019.

MARSARO JÚNIOR, A. L. et al. First report of Lecanodiaspis dendrobii Douglas, 1892 (Hemiptera: Lecanodiaspididae) and the associated parasitoid Cephaleta sp. (Hymenoptera: Pteromalidae) in Brazil. Revista Brasileira de Biologia, Uberlândia, v. 76, n. 1, p. 250-255, 2016.

MILLER, D. R.; RUNG, A.; PARIKH, G. Scale Insects, edition 2, a tool for the identification of potential pest scales at U.S.A. ports-of-entry (Hemiptera, Sternorrhyncha, Coccoidea). ZooKeys, Sofia, v. 431, n. 1, p. 61-78, 2014.

NASCIMENTO, D. A. et al. Ocorrência dos ácaros Mononychellus sp. e Oligonychus sp. em mogno africano (Khaya ivorensis) no Brasil. Nativa, Sinop, v. 4, n. 1, p. 58-60, 2016.

PERES-FILHO, O. et al. Maconellicoccus hirsutus (Green) Register in Teak Forest Stands in the Mato Grosso State, Brazil. Floresta e Ambiente, Seropédica, v. 24, p. e20150157, 2017.

PEREZ, J. et al. Leaves from grafted meliaceae species affect survival and performance of Hypsipyla grandella (Zeller) (Lepidoptera: Pyralidae) larvae. Journal of Pest Science, Berlim, v. 83, n. 2, p. 95-104, 2010.

PINHEIRO, A. L. et al. Ecologia, silvicultura e tecnologia de utilização dos mognos-africanos (Khaya spp.). Viçosa, MG: Sociedade Brasileira de Agrossilvicultura, 2011. 102 p.

RIBEIRO, A.; FERRAZ FILHO, A. C.; SCOLFORO, J. R. S. African Mahogany (Khaya spp.) Cultivation and the Increase of the Activity in Brazil. Floresta e Ambiente, Seropédica, v. 24, p. e00076814, 2017.

SANTOS, G. P. et al. Pragas do eucalipto. Informe Agropecuário, Belo Horizonte, v. 29, n. 242, p. 43-64, 2008.

SANTOS, R. S.; PERONTI, A. L. B. G. Ocorrência de Phenacoccus solenopsis Tinsley (Hemiptera: Pseudococcidae) em quiabeiro no estado do Acre. EntomoBrasilis, [s. l.], v. 10, n. 2, p. 135-138, 2017.

ZANETTI, R. et al. First report of Hypsipyla grandella (Lepidoptera: Pyralidae) on African mahogany Khaya ivorensis. Scientia Agricola, Piracicaba, v. 74, n. 6, p. 492-494, 2017. 